\title{
The head and body lice of humans are genetically distinct (Insecta: Phthiraptera, Pediculidae): evidence from double infestations
}

\author{
NP Leo ${ }^{1}$, JM Hughes ${ }^{2}$, X Yang ${ }^{3}$, SKS Poudel ${ }^{4}$, WG Brogdon ${ }^{5}$ and SC Barker ${ }^{1,6}$ \\ ${ }^{1}$ Department of Microbiology and Parasitology, University of Queensland, Brisbane 4072, Australia; ${ }^{2}$ Australian School of \\ Environmental Studies, Griffith University, Brisbane 4111, Australia; ${ }^{3}$ Animal Medical Department, Inner Mongolia Agricultural \\ University, Huhehot 010018, China; ${ }^{4}$ Department of Science, Janapriya Multiple Campus, Pokhara, Nepal; ${ }^{5}$ Division of Parasitic \\ Diseases, NCID, CDC Atlanta, GA, USA; ' Institute of Molecular Biosciences, University of Queensland, Brisbane 4072, Australia
}

\begin{abstract}
Little is known about the population genetics of the louse infestations of humans. We used microsatellite DNA to study 11 double infestations, that is, hosts infested with head lice and body lice simultaneously. We tested for population structure on a host, and for population structure among seven hosts that shared sleeping quarters. We also sought evidence of migration among louse populations. Our results showed that: (i) the head and body lice on these individual
\end{abstract}

hosts were two genetically distinct populations; (ii) each host had their own populations of head and body lice that were genetically distinct to those on other hosts; and (iii) lice had migrated from head to head, and from body to body, but not between heads and bodies. Our results indicate that head and body lice are separate species.

Heredity (2005) 95, 34-40. doi:10.1038/sj.hdy.6800663

Published online 1 June 2005

Keywords: Pediculus; head lice; body lice; transmission; population structure; double infestation

\section{Introduction}

There are two forms of Pediculus that infest humans: head lice that live and lay their eggs on the hair of the head, and body lice that live and lay their eggs in clothes. Body lice probably evolved from head lice when humans began to wear clothes; a recent study attempted to date the origin of clothes from the time of divergence of head and body lice (Kittler et al, 2003). However, whether head and body lice interbreed in nature is still unresolved. Busvine (1978) used morphological markers to test if head lice and body lice interbreed in nature. When head and body lice interbreed, their offspring have morphology that is intermediate between that of head and body lice (Busvine, 1948). Busvine (1978) did not find lice with intermediate morphology on hosts that were infested with both head and body lice. Despite Busvine's results, head and body lice are often considered to be subspecies of Pediculus humanus (eg Durden and Musser, 1994). Indeed, evidence from mitochondrial DNA and nuclear DNA reveals that head lice and body lice are not reciprocally monophyletic (Leo et al, 2002; Kittler et al, 2003; Yong et al, 2003; Reed et al, 2004). If head and body lice speciated recently, however, they would almost certainly not be reciprocally monophyletic. This is because there would have been insufficient time for lineage sorting to result in two reciprocally monophyletic lineages.

Correspondence: NP Leo, Department of Microbiology and Parasitology, University of Queensland, Cooper Road, St Lucia, Brisbane 4072, Australia. E-mail: nleo@uqconnect.net

Received 31 March 2004; accepted 7 February 2005; published online 1 June 2005
We used microsatellite markers to discover if head lice and body lice on a host were one panmictic population or two genetically distinct populations, how many populations of lice infested the seven hosts that shared sleeping quarters, and to identify lice that had migrated between the head and body on a host, or from host to host. We defined a population as a group of individuals that mate at random (otherwise known as Mendelian populations or demes).

\section{Materials and methods}

\section{Sample collection}

Lice were preserved in $100 \%$ ethanol or $20 \%$ dimethylsulfoxide (DMSO) in a saturated sodium chloride solution. We studied 443 lice from 11 double infestations from China and Nepal (Table 1). These included two sisters from China that shared some clothes, a bed and a haircomb, and five male street children from a recycling yard in Nepal that all slept in the same shed.

\section{DNA extraction, PCR and electrophoresis}

DNA was extracted from whole lice with a solution of $5 \%$ chelex beads (Bio-Rad, CA, USA) in $1 \times$ TE buffer. DNA from each louse was amplified by PCR at five loci with primers (Table 2). The five loci were found in the genomic libraries of body lice and head lice. Three loci contained dinucleotide repeats: microsatellite locus 7 (ML7), ML8, and ML9. The other two loci contained trinucleotide repeats: ML10 and ML21 (Table 2). Reverse primers were labelled with a fluorescent Hex label (Proligo, Germany) to allow visualisation of PCR product. The Red Hot Taq polymerase kit from AB Gene 
Table 1 Details of the 11 double infestations of head lice and body lice (443 lice) studied

\begin{tabular}{|c|c|c|c|c|c|}
\hline Country & Location & Host ID & $\begin{array}{l}\text { Site on } \\
\text { host }\end{array}$ & $\begin{array}{l}\text { Sample size total (number of } \\
\text { lice from each lifestage) }\end{array}$ & Other information \\
\hline \multirow[t]{7}{*}{ China } & \multirow{5}{*}{$\begin{array}{l}\text { Remote village near the } \\
\text { capital, Huhehot, in inner } \\
\text { Mongolia Province }\end{array}$} & B1417 & $\begin{array}{l}\text { Head } \\
\text { Body }\end{array}$ & $\begin{array}{l}16(4 \mathrm{~F}, 8 \mathrm{M}, 2 \mathrm{~N} 3,2 \mathrm{E}) \\
21(1 \mathrm{~F}, 4 \mathrm{M}, 2 \mathrm{~N} 3,14 \mathrm{~N} 2)\end{array}$ & Sister of host B1418 \\
\hline & & B1418 & Head & $9(3 \mathrm{~F}, 4 \mathrm{M}, 2 \mathrm{E})$ & \multirow[t]{2}{*}{ Sister of host B1417 } \\
\hline & & & Body & $14(3 \mathrm{~F}, 2 \mathrm{M}, 6 \mathrm{~N} 3,3 \mathrm{~N} 2)$ & \\
\hline & & B1457 & Head & $10(3 \mathrm{~F}, 6 \mathrm{M}, 1 \mathrm{~N} 2)$ & \multirow{4}{*}{$\begin{array}{l}\text { Sample taken } 9 \text { months } \\
\text { after B1417 and B1418 } \\
\text { from one of the two sisters }\end{array}$} \\
\hline & & & Body & $27(11 \mathrm{~F}, 6 \mathrm{M}, 9 \mathrm{~N} 3,1 \mathrm{~N} 2)$ & \\
\hline & Village near the town & B1531 & Head & $28(9 \mathrm{~F}, 3 \mathrm{M}, 3 \mathrm{~N} 3,7 \mathrm{~N} 2,5 \mathrm{~N} 1,1 \mathrm{E})$ & \\
\hline & $\begin{array}{l}\text { Dongshen, in Inner } \\
\text { Mongolia Province }\end{array}$ & & Body & $34(2 \mathrm{~F}, 12 \mathrm{M}, 17 \mathrm{~N} 3,3 \mathrm{~N} 2)$ & \\
\hline \multirow[t]{14}{*}{ Nepal } & Kathmandu & B2002 & Head & $48(9 \mathrm{~F}, 6 \mathrm{M}, 5 \mathrm{~N} 3,28 \mathrm{~N} 2)$ & \\
\hline & & & Body & $48(21 \mathrm{~F}, 27 \mathrm{M})$ & \\
\hline & Pokhara, recycling yard A & B2006 & Head & 48 (12F, 3M, 33N3) & \\
\hline & & & Body & $29(10 \mathrm{~F}, 5 \mathrm{M}, 5 \mathrm{~N} 3,6 \mathrm{~N} 2,1 \mathrm{~N} 1,2 \mathrm{E})$ & \\
\hline & \multirow[t]{10}{*}{ Pokhara, recycling yard B } & B2009 & Head & $18(1 \mathrm{~F}, 1 \mathrm{~N} 3,6 \mathrm{~N} 2,1 \mathrm{~N} 1,9 \mathrm{E})$ & \multirow{10}{*}{$\begin{array}{l}\text { Hosts in recycling yard B } \\
\text { shared sleeping quarters - } \\
\text { all were male street- } \\
\text { children }\end{array}$} \\
\hline & & & Body & $16(2 \mathrm{M}, 12 \mathrm{~N} 3,1 \mathrm{~N} 2,1 \mathrm{~N} 1)$ & \\
\hline & & B2010 & Head & $8(2 \mathrm{~F}, 2 \mathrm{~N} 1,4 \mathrm{E})$ & \\
\hline & & & Body & $5(2 \mathrm{~F}, 2 \mathrm{M}, 1 \mathrm{~N} 3)$ & \\
\hline & & B2011 & Head & $10(1 \mathrm{~F}, 9 \mathrm{E})$ & \\
\hline & & & Body & $14(3 \mathrm{~F}, 2 \mathrm{M}, 3 \mathrm{~N} 3,5 \mathrm{~N} 2,1 \mathrm{~N} 1)$ & \\
\hline & & B2012 & Head & $4(1 \mathrm{~F}, 1 \mathrm{~N} 3,1 \mathrm{~N} 1,1 \mathrm{E})$ & \\
\hline & & & Body & $6(3 \mathrm{M}, 1 \mathrm{~N} 3,2 \mathrm{~N} 1)$ & \\
\hline & & B2013 & Head & $14(6 \mathrm{~F}, 1 \mathrm{M}, 1 \mathrm{~N} 3,1 \mathrm{~N} 2,5 \mathrm{E})$ & \\
\hline & & & Body & $16(2 \mathrm{M}, 2 \mathrm{~N} 3,9 \mathrm{~N} 2,3 \mathrm{~N} 1)$ & \\
\hline
\end{tabular}

Note that hosts B1417 and B1418 each had one head louse and one body louse that were also in Leo et al (2002). F=female; M=male; $\mathrm{N} 3=3$ rd stage nymph; N2 = 2nd stage nymph; N1 =1st stage nymph; $\mathrm{E}=$ egg; ID = identification.

Table 2 Primers used to amplify the five microsatellite loci used in this study.

\begin{tabular}{llll}
\hline Locus name & Primer name & Primer sequence 5'-3' & Repeat motif \\
\hline ML7 & ML7F & AAG CTT TCT GCT ACA TT & AG \\
& ML7RH & GGG AAG TGG CGT AAT CGT T & TC \\
ML8 & ML8F & AAA CGT TCG AAT GGG ACT TGA AC & TC \\
ML9 & ML9F & CCC ACA CAT AGC CGC CAT T & TTA \\
ML10 & ML9RH & TCC ATC ATC CAT CGT TTC GA & ATT \\
ML21 & ML10F & CCG ATT CTC CTT ATT CAT TCG C & GCC AAG GTC TAA TTA TCC \\
& ML10RH & GAA CGA GAA GAT GCG TAA ACG & \\
\hline
\end{tabular}

$\mathrm{F}=$ forward primer; $\mathrm{R}=$ reverse primer; $\mathrm{H}=$ Hex-labelled.

(Epsom, UK) was used to amplify DNA. The following temperature cycle was used: one cycle of $94^{\circ} \mathrm{C}$ for $3 \mathrm{~min}$; 35 cycles of $93^{\circ} \mathrm{C}$ for $30 \mathrm{~s}, 51^{\circ} \mathrm{C}$ for $30 \mathrm{~s}$ (except ML10 at $42^{\circ} \mathrm{C}$ ), $72^{\circ} \mathrm{C}$ for $30 \mathrm{~s}$; one cycle of $72^{\circ} \mathrm{C}$ for $7 \mathrm{~min}$.

PCR products were electrophoresed at $1200 \mathrm{~V}$ for $90 \mathrm{~min}$ on a 5\% acrylamide denaturing gel with an automated DNA fragment analyser (Corbett Research Gel Scan V7.2.6) at $40^{\circ} \mathrm{C}$. Genescan TAMRA 350 size standard (PE Applied Biosystems, CA, USA) was used in conjunction with samples of known size to provide points of reference and ensure consistent scoring across gels. Gels were scored with the software package ONE-Dscan (Scanalytics, VA, USA), and double-checked by eye.
Tests for Hardy-Weinberg equilibrium (HWE)

GENEPOP on the web (http://wbiomed.curtin. edu.au/ genepop/index.html) (Raymond and Rousset, 1995) was used to run an exact test for HWE at each locus for head lice and body lice from each host. The Markov Chain parameters were set at 1000 dememorizations, 300 batches, and 2000 iterations per batch. Results were considered statistically significant if $P<0.05$ after sequential Bonferroni correction (110 tests - five loci for each of 22 populations). We also used GENEPOP to calculate the inbreeding coefficients $\left(\mathrm{F}_{\mathrm{IS}}\right)$ for head and body lice from each host at each locus. The proportion of observed and expected heterozygotes was calculated in ARLEQUIN 2.000 (Schneider et al, 2000). 
Estimating the number of louse populations on and among hosts

To determine if the head lice and body lice on a host were one or two populations, we compared the genotype frequencies of head and body lice from each host with a G-based exact test in GENEPOP. We repeated this for head and body lice pooled from hosts that shared sleeping quarters: B1417 and B1418 (sisters), and B2009-B2013 (five street children that shared a shelter).

We used the program ARLEQUIN to execute hierarchical AMOVAs. Gametic phase was listed as unknown, and 10000 permutations were run to test the significance of fixation indices. We ran two AMOVAs each for lice on the two sisters, and for lice on the five hosts from recycling yard B: (i) lice were grouped by host, with head and body lice from the same host as populations within a group, and (ii) lice were grouped by type (head or body), with populations of head lice in one group and populations of body lice in the other group.

The program STRUCTURE 2.0 (Pritchard et al, 2000) uses Hardy-Weinberg proportions and linkage equilibrium to construct likely populations from a given sample of individuals and generates a posterior probability for those populations. We used STRUCTURE to generate posterior probabilities for 1-5 populations of lice on the two sisters from China, B1417 and B1418 (two runs of 150000 burn-in and $1000000 \mathrm{MCMC}$ repetitions). Lice from five hosts from recycling yard B were tested for 1-5 and 10 populations (60000 burn-in and 1000000 repetitions). We also ran simulations for lice from each host for two populations, to allow comparison with assignment of lice (see below).

\section{Assignment of lice to populations}

For each host, we used STRUCTURE to generate posterior probabilities that each louse belonged to the other collection site (head or body), or had a parent or grandparent from the other site. The probability that an individual was a migrant to a collection site, or had a migrant ancestor $(v)$ was set at 0.05 . When the posterior probability of a louse to its collection site was $<0.8$, we suspected that the louse was a migrant. The value of 0.8 was chosen arbitrarily to aid in the identification of potential migrants. We tested suspected migrants again at $v=0.1$, to check for sensitivity of the posterior probability to changes in $v$, which may indicate that STRUCTURE does not have enough information for a confident assignment (Pritchard et al, 2000). An arbitrary maximum of 0.1 variation between runs was chosen; lice with posterior probabilities that exceeded this were considered too unreliable for strong conclusions to be drawn about their origins.

\section{Results and discussion}

\section{Were lice in HWE?}

Probability tests showed that 17 of 110 tests were significantly out of HWE (Supplementary Table 1). Only B1457 body lice, B1531 head lice and B1531 body lice had three or more loci out of HWE. Our results indicate that certain populations rather than certain loci were consistently out of HWE. All but one of these tests had positive $\mathrm{F}_{\mathrm{IS}}$ values, which indicates a deficit of hetero- zygotes compared to that expected for HWE. This heterozygote deficit could be explained by null alleles or by population subdivision. We cannot discount either of these possibilities, but our results showed that certain populations were out of HWE rather than certain loci, so it is most likely that the heterozygote deficit is a population-specific phenomenon. A deficit of heterozygotes in louse populations could also occur if the population was recently founded by just a few lice. Doubtless some infestations are the progeny of a single pair of lice.

\section{Were the head and body lice on a host one panmictic population?}

Our analyses showed that the head lice and body lice on a host were two populations. G-based exact tests that compared head and body lice from each host showed that lice from all hosts, except B2010 and B2012, were significantly different at three or more of the five loci (Table 3). The lack of genetic differentiation between head and body lice on these two hosts was probably due to the small samples of lice: eight head lice and five body lice from B2010, and four head lice and six body lice from B2012. Indeed, these were the two smallest samples in our study (see Table 1).

With no information on collection site (head or body), STRUCTURE assigned more than $80 \%$ of lice on hosts B1417, B1418, B1457, B2002 and B2009 to two populations that corresponded to head lice and body lice. However, hosts B1531, B2006, B2010, B2011, B2012 and B2013 had $50-100 \%$ of lice with roughly equal probability of belonging to each of two populations. This might indicate a lack of population structure (ie head and body lice from the same host were from one panmictic population), but this is unlikely since the G-based exact tests showed significant differences between head and body lice for nine of the 11 hosts. The exceptions were hosts B2010 and B2012, both of which were represented by small samples of lice (Table 1). A more likely explanation is that STRUCTURE did not have enough information to distinguish between the populations on these six hosts (too few loci or small sample size), or that the assumptions STRUCTURE uses to construct populations (HWE and linkage equilibrium), were violated. Overall, the results indicated that head and body lice on

Table 3 Tests for genotypic differentiation between the head lice and the body lice of 11 hosts at five loci, executed in GENEPOP (1000 dememorizations, 100 batches and 1000 repetitions per batch)

\begin{tabular}{lccccc}
\hline Host & $M L 7$ & $M L 8$ & ML9 & ML10 & ML21 \\
\hline B1417 & $*$ & $*$ & $*$ & $*$ & $*$ \\
B1418 & $*$ & $*$ & NS & $*$ & $*$ \\
B1457 & $*$ & $*$ & $*$ & $*$ & $*$ \\
B1531 & $*$ & $*$ & $*$ & $*$ & $*$ \\
B2002 & $*$ & $*$ & $*$ & $*$ & $*$ \\
B2006 & $*$ & $*$ & $*$ & $*$ & $*$ \\
B2009 & $*$ & NS & $*$ & $*$ & $*$ \\
B2010 & $*$ & NS & NI & NI & NS \\
B2011 & $*$ & NS & $*$ & $*$ & $*$ \\
B2012 & NS & NS & NI & NS & NS \\
B2013 & $*$ & NS & $*$ & $*$ & NS \\
\hline
\end{tabular}

* = Significant $(P<0.05) ; \mathrm{NS}=$ nonsignificant $(P<0.05) ; \mathrm{NI}=$ not enough information was available to perform test. 
a host were not one panmictic population. Rather, head lice were genetically distinct to the body lice on the same host, so from here on we refer to them as different populations.

\section{How many populations were present on the seven hosts that shared sleeping quarters?}

We had two samples of lice from hosts that shared sleeping quarters: one sample from the two sisters, and the other from five street-children from recycling yard B. When we pooled lice from hosts that shared sleeping quarters, the genotype frequencies of head lice and body lice were significantly different $(P=0.00000$ at all loci for sisters and for recycling yard B). However, hierarchical AMOVAs showed that head lice and body lice pooled from these sleeping quarters did not form two panmictic populations. The AMOVA detected significant differences among lice from different hosts within head lice, and within body lice. For the two sisters, the difference between head lice and body lice $\left(\mathrm{F}_{\mathrm{CT}}=0.26733\right.$, not significant) was greater than between populations within head lice or within body lice $\left(\mathrm{F}_{\mathrm{SC}}=0.04807, P=0.00000\right)$. The results from the five hosts of recycling yard $B$ also showed greater differentiation between head and body lice $\left(\mathrm{F}_{\mathrm{CT}}=0.08174, P=0.00703\right)$ than among populations from the different hosts $\left(F_{\mathrm{SC}}=0.04807, P=0.00000\right)$. Therefore, the results indicate that differentiation between head and body lice was greater than the differentiation among lice from different hosts, and that there were four populations of lice on the two sisters, that is, each sister had populations of head lice and body lice that were genetically distinct from the lice of the other sister.

With no information on collection site (head or body) from the two sisters, the program STRUCTURE gave the highest posterior probability for four populations being present (instead of 1, 2, 3, or 5 populations; Figure 1a). All head lice from the two sisters were assigned to two populations, but these did not correspond to the two sisters. Likewise, all but one of the body lice were assigned to two populations that did not correspond to the two sisters. When STRUCTURE assigned lice from the two sisters to two populations, head lice formed one population and body lice the other, but one body louse was assigned to the head lice (Figure 1b). So STRUCTURE was able to distinguish between head and body lice, but not between head lice from different sisters, nor between body lice from different sisters. In the case of the five hosts from recycling yard B, STRUCTURE did not distinguish head and body lice (two populations), or head and body lice from each host (10 populations). One explanation for this result is that STRUCTURE did not have enough information to distinguish between populations that were more similar genetically (see results of hierarchical AMOVAs above).

Detection of lice that had migrated between populations of head lice and body lice on a host

We tested for migrants between the populations of head lice and body lice on each host with STRUCTURE. Hosts with lice that had posterior probabilities less than 0.8 for belonging to their site of collection were analysed again at $v=0.1$ (Supplementary Table 2). Of 14 lice that were tested again at $v=0.1$, the posterior probabilities of only four lice varied by less than 0.1 between runs of STRUCTURE: two body lice from host B2009 (lice 1 and 2), one body louse from host B2006 (louse 3), and one head louse from B2013 (louse 4). The posterior probabilities indicated that i) the body louse from B2006 may have been descended from head lice $(0.567$ probability of no migrant ancestry), and that the two body lice from B2009 were migrants from the head louse population on the same host, B2009. The head louse from B2013 was still assigned to the head lice population both times (probability of 0.676 and higher). However, this analysis did not have lice from all possible sources of migrants (eg other street-children from recycling yard B), which may have affected the posterior probabilities of some lice. This is discussed further in the next section.

\section{Migration of lice among the hosts that shared sleeping quarters}

We informed STRUCTURE of the collection sites of lice from the two sisters. No migrants were detected when lice were assigned to two populations of head lice and body lice (Figure 1c, posterior probabilities not shown). However, when we informed STRUCTURE of four collection sites (head and body lice from each sister), there were four lice with posterior probabilities of less than 0.8 for belonging to the site from which they were collected (Figure 1d, Supplementary Table 3). One head louse from B1418 was assigned to the head louse population of B1417 (louse 15); the other three head lice had posterior probabilities that fluctuated by more than 0.1 but most likely these three head lice belonged to the population from which they were collected. These results concur with the results of the hierarchical AMOVA for the presence of four populations, since if the head lice on the two sisters were one panmictic population, we would not expect to find migrants.

We then tested for migrants among the 111 lice from Recycling yard B. We informed STRUCTURE of 10 populations (head lice and body lice on five hosts). Three head lice and eight body lice had posterior probabilities of less than 0.8 for belonging to the population from which they were collected (Supplementary Table 4). Four of these lice had scores that varied by less than 0.1 between runs at $v=0.05$ and 0.1 : louse 1 from the body of B2009 had a moderate probability (0.689) of belonging to B2011 body lice; one body louse from B2013 (louse 18) had a high probability (0.959) of being an migrant from the body louse population of B2009; and body lice 2 and 5 from host B2009 had low probabilities ( 0.26 or less) for belonging to other populations of body lice. It is noteworthy that body louse 1 and 2 from host B2009 were assigned to other populations of body lice, but in the analyses of lice from host B2009 only, they were assigned to the head louse population (Supplementary Table 2). This discrepancy is probably due to the absence of potential sources of migrants, for example, lice from other friends.

What do these analyses tell us about transmission of lice? If we consider only lice from multiple hosts, with posterior probabilities that varied by less than 0.1 between runs, our results are evidence for movement of lice among hosts, but not between heads and bodies (Supplementary Tables 3 and 4). Three lice (lice 1, 15 and 

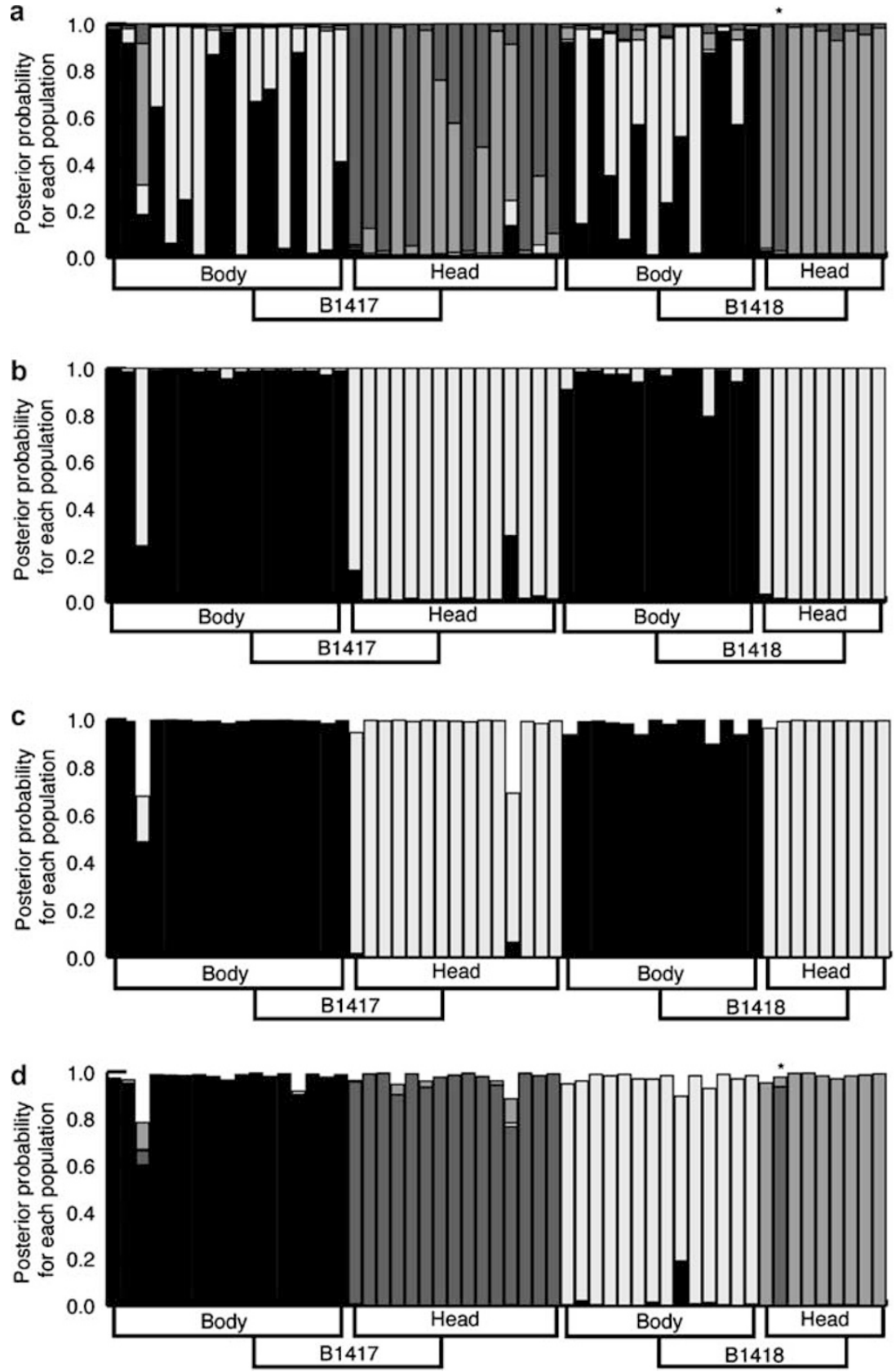

Figure 1 Posterior probabilities of lice from two sisters, B1417 and B1418, for belonging to: (a) four populations and no prior information on collection site; (b) two populations and no prior information on collection site; (c) two populations and known collection site (head and body); (d) four populations and known collection site (head, body and sister). Populations are represented by different shades. Note that the probabilities in (c and $\mathbf{d}$ ) do not always sum to one since there were small probabilities associated with having migrant ancestry that are not shown. *louse 15.

18) were likely first-generation migrants. The other two lice (lice 2 and 5) had only low probabilities for belonging to other body lice populations; this might indicate that a larger sample size of loci or hosts is needed, or that these lice had ancestors from all of these body lice populations. Buxton (1947) thought that most transmission of body lice was by adults: he observed that adult body lice are more mobile than nymphs and that new infestations generally have more adults than nymphs. Our results revealed two body louse nymphs that were probably first-generation migrants from body louse populations of other hosts (lice 1 and 18, 2nd and 3rd stage nymphs, respectively). So, we found body lice nymphs were as likely to be migrants as were adult body lice; however, a larger sample size would be needed to properly test Buxton's hypothesis (Buxton, 1947).

In the case of head lice, Maunder (1983) suggested that head lice start to actively disperse after their first mating whereas nymphs transfer much less readily. Maunder described head lice on different hosts as 'an actively intermingling community inhabiting an archipelago'. He suggested that in one day a louse might visit several 
heads. Our results indicate that head lice migrated from one host to another but not as frequently as Maunder (1983) suggested, since we found only one female louse that had migrated from one head to another head. Indeed, we detected more body lice migrants than head lice migrants, although this could be biased by host behavior. For example, no head lice migrants were found among the male street-children, possibly because they did not share a comb like the sisters, and so their head lice had fewer opportunities to migrate to a different head. The effect of host behavior on the frequency of transmission could be considered in future studies.

\section{Are populations of head and body lice kept separate by behaviour?}

There are reports that head lice placed on the clothes of a person returned to the head hair immediately (Sikora, 1917 in Howlett, 1918; Busvine, 1978). This behavioural separation (site-preference) of head lice and body lice could explain why head lice and body lice that infest a person simultaneously are two genetically distinct populations. A study by Keilin and Nuttall (1919) showed that head and body lice may be genetically incompatible; the hybrid offspring of head and body lice bred in the laboratory were more likely to be hermaphrodites than the offspring of 'wild lice'. If head and body lice are kept separate by genetic incompatibility, one would expect to find some hybrid lice from hosts that are doubly infested. Instead, our results found that no lice had migrated between populations of head and body lice; no head lice had any body lice parents or grandparents, and no body lice had any head lice parents or grandparents (Supplementary Tables 3 and 4). Rather, all four lice had apparently migrated from a head to a head or a body to a body, with no evidence for hybrids or hybrid ancestors. Our results are consistent with the only other study of double infestations (Busvine, 1978). This provides evidence that head lice and body lice are kept separate genetically, by ecological and/or behavioural factors, and not by genetic incompatibility.

\section{Conclusion}

Our analyses show that the head lice and body lice we studied had not interbred, and that each host had populations of head and body lice that were genetically distinct to those of other hosts. Lice had migrated from head to head, and from body to body, but not between heads and bodies.

Recent studies of head lice and body lice with molecular markers indicate that body lice evolved recently (Kittler et al, 2003), and that head and body lice are not reciprocally monophyletic lineages (Leo et al, 2002; Yong et al, 2003; Reed et al, 2004). Yong et al (2003) reported putative phylogenetic markers ('signature mutations') that distinguish head and body lice from countries outside sub-Saharan Africa, but we found a Russian head louse from Yong et al (2003) that had four phylogenetic markers for head lice, and nine for body lice. None of the previous studies could reveal if head and body lice are currently interbreeding.

The present study revealed that the head and body lice of double infestations do not interbreed. This concurs with the results from the only other study of double infestations (Busvine, 1978). Busvine (1978) used morphological markers to examine lice from Ethiopia, Africa. We used genetic markers to examine lice from Nepal and China. So Busvine (1978) and the present study used two different methods and came to the same conclusion: that head and body lice do not interbreed in nature. At first glance, this seems to contradict the results from phylogenetic studies that show that head and body lice are not reciprocally monophyletic (Leo et al, 2002; Kittler et al, 2003; Yong et al, 2003; Reed et al, 2004). We suggest that incomplete lineage sorting explains why head and body lice were not reciprocally monophyletic even when they do not interbreed in nature (see Page and Holmes, 1998 for an explanation of lineage sorting). If head and body lice are separate species, the names that should be used are $P$. capitis de Geer, 1778 for head lice, and P. humanus Linnaeus, 1758 for body lice (see Ferris, 1951; Hemming, 1958).

\section{Acknowledgements}

We thank Dr Bruce Hayes from Patan Hospital, Kathmandu, for helping SCB collect lice, Gena Groner and Robert A Wirtz from the CDC for creating the microsatellite library, and Jing Ma and David Gopurenko from the Australian School of Environmental Studies, Griffith University for technical advice on the microsatellite work.

\section{References}

Busvine JR (1948). The 'head' and 'body' races of Pediculus humanus L. Parasitology 39: 1-16.

Busvine JR (1978). Evidence from double infestations for the specific status of human head and body lice (Anoplura). Syst Entomol 3: 1-8.

Buxton PA (1947). The Louse: An Account of the Lice Which Infest Man, their Medical Importance and Control, 2nd edn. Edward Arnold \& Co.: London.

Durden LA, Musser GG (1994). The sucking lice (Insecta: Anoplura) of the world: a taxonomic checklist with records of mammalian hosts and geographical distributions. Bull Am Mus Nat Hist 1-90.

Ferris GF (1951). The Sucking Lice. The Pacific Coast Entomological Society: San Francisco.

Hemming F (1958). Official List of Generic Names in Zoology. First Installment: Names 1-1274. International Commission on Zoological Nomenclature: London.

Howlett FM (1918). Notes on head- and body-lice and upon temperature reactions of lice and mosquitoes. Parasitology $\mathbf{1 0}$ 186-188.

Keilin D, Nuttall GHF (1919). Hermaphroditism and other abnormalities in Pediculus humanus. Parasitology 11: 279-328.

Kittler R, Kayser M, Stoneking M (2003). Molecular evolution of Pediculus humanus and the origin of clothing. Curr Biol 13: 1414-1417.

Leo NP, Campbell NJH, Yang X, Mumcuoglu KY, Barker SC (2002). Evidence from mitochondrial DNA that the head lice and the body lice of humans (Phthiraptera: Pediculidae) are conspecific. J Med Entomol 39: 662-666.

Maunder JW (1983). The appreciation of lice. Proc Roy Inst G B 55: 1-31.

Page RDM, Holmes EC (1998). Molecular Evolution: A Phylogenetic Approach. Blackwell Science: Oxford, Malden, MA.

Pritchard JK, Stephens M, Donnelly P (2000). Inference of population structure using multilocus genotype data. Genetics 155: 945-959. 
Raymond M, Rousset F (1995). GENEPOP version 1.2: population genetics software for exact tests and ecumenicism. J Hered 86: 248-249.

Reed DL, Smith VS, Hammond SL, Rogers AR, Clayton DH (2004). Genetic analysis of lice supports direct contact between modern and archaic humans. PLoS Biol 2: e340.
Schneider S, Roessli D, Excoffier L (2000). Arlequin Ver 2000. A Software for Population Genetics Data Analysis. Genetics and Biometry Lab, University of Geneva: Switzerland.

Yong Z, Fournier P-E, Rydkina E, Raoult D (2003). The geographical separation of human lice preceded that of Pediculus humanus capitis and Pediculus humanus humanus. $C R$ Biol 326: 565-574.

Supplementary Information accompanies the paper on the Heredity web site (http://www.nature.com/hdy) 